\title{
Diagnostics and characterization of nanodust and nanodusty plasmas
}

\author{
Franko Greiner ${ }^{1}$, Andrè Melzer ${ }^{2}$, Benjamin Tadsen ${ }^{1}$, Sebastian Groth ${ }^{1}$, Carsten Killer ${ }^{3}$, Florian Kirchschlager ${ }^{4}, 6$, \\ Frank Wieben ${ }^{1}$, Iris Pilch ${ }^{5}$, Harald Krüger ${ }^{2}$, Dietmar Block ${ }^{1}$, Alexander Piel ${ }^{1}$, and Sebastian Wolf ${ }^{4}$ \\ 1 Institut für Experimentelle und Angewandte Physik, Christian-Albrechts-Universität zu Kiel, 24098 Kiel, Germany \\ 2 Institut für Physik, Ernst-Moritz-Arndt-Universität, D-17489 Greifswald, Germany \\ 3 Max-Planck Institut für Plasmaphysik, EURATOM Association, D-17491 Greifswald, Germany \\ ${ }^{4}$ Institut für Theoretische Physik und Astrophysik, Christian-Albrechts-Universität zu Kiel, 24098 Kiel, Germany \\ 5 Thin Film Division, IFM-Materials Physics, Linköping University, SE-581 83 Linköping, Sweden \\ 6 Department of Physics and Astronomy, University College London, Gower Street, London WC1E 6BT, UK
}

Received: date / Revised version: date

\begin{abstract}
Plasmas growing or containing nanometric dust particles are widely used and proposed in plasma technological applications for production of nano-crystals and surface deposition. Here, we give a compact review of in-situ methods for the diagnostics of nanodust and nanodusty plasmas, which have been developed in the framework of the SFB-TR24 to fully characterize these systems. The methods include kinetic Mie ellipsometry, angular-resolved Mie scattering, and 2D imaging Mie ellipsometry to get information about particle growth processes, particle sizes and particle size distributions. There, also the role of multiple scattering events is analyzed using radiative transfer simulations. Computed tomography and Abel inversion techniques to get the 3D dust density profiles of the particle cloud will be presented. Diagnostics of the dust dynamics yields fundamental dust and plasma properties like particle charges and electron and ion densities. Since nanodusty plasmas usually form dense dust clouds electron depletion (Havnes effect) is found to be significant.
\end{abstract}

PACS. 52.25.Os 52.25.Vy 52.27.Lw 52.70.Kz 52.80.Pi 81.15.Gh

\section{Introduction}

A nanodusty plasma is a plasma that contains ions, electrons and nanoparticles, i.e. solid particles in the size range between a few and a few hundred nanometers. The properties of nanodusty plasmas are decisively different from dust-free and dusty plasmas that contain micrometer-sized dust. This is due to the fact that in nanodusty plasmas the dust density $n_{\mathrm{d}}$ usually is so high that the quasineutrality condition is strongly affected by the dust charge density. This is best described by the Havnes parameter [1]

$$
P=4 \pi \epsilon_{0} a \frac{k_{\mathrm{B}} T_{\mathrm{e}}}{e^{2}} \frac{n_{\mathrm{d}}}{n_{\mathrm{i}}},
$$

giving the dust charge density in units of the ion density $n_{\mathrm{i}}$. Here, $\epsilon_{0}$ is the permittivity of free space, $a$ the mean radius of the nanoparticles, $T_{\mathrm{e}}$ the electron temperature, $k_{\mathrm{B}}$ the Boltzmann constant and $e$ the elementary electric charge. In plasmas with low dust density $(P<1)$ the plasma parameters are not strongly affected by the presence of the dust. In nanodusty plasmas, however, generally the Havnes parameter exceeds unity $(P>1)$ indicating that nearly all electrons reside on the dust and the free electrons are depleted. Figure 1 shows the corresponding change in the plasma and dust potential due to the Havnes effect. At a low dust density $(P \ll 1)$ the plasma potential is that of a dust-free plasma, the dust acquires a floating potential $\phi_{\mathrm{f}}$ expected from undisturbed ion and electron currents to the particle. At $P \approx 1$ the free electron density is not sufficient to charge the dust to its free floating potential and correspondingly the plasma potential in the cloud $\left(\Phi_{\mathrm{C}}\right)$ drops, until at very dense dust clouds $(P \gg 1)$ the cloud and dust potential become equal and essentially no free electrons are left. We will see, that nanodusty plasma can reach states with a Havnes parameter of $P=50$.

A high dust content leads to problems with classical plasma diagnostics like Langmuir probes, optical emission spectroscopy or laser-based techniques such as laser induced fluorescence. Hence, the key to nanodusty plasma diagnostics is the measurement of particle size and particle size distributions, since the dust size determines the dust charging. Further, techniques for the direct determination of the spatially resolved dust density will be described and, finally, methods will be presented to retrieve the plasma parameters from the dust dynamics.

In Sec. 2, we will describe experimental techniques to create nanodusty plasmas where we will focus on parti- 


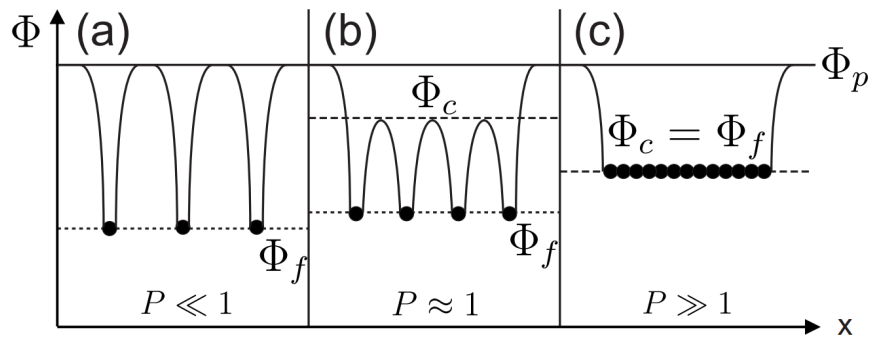

Fig. 1. Transition from "dust in a plasma" $(P \ll 1)$ to a "dusty" plasma $(P \gg 1)$, from [2], see text for details.

cle formation in reactive argon-acetylene plasmas. In the following sections, we give a short introduction to the different methods used to characterize nanodusty plasmas. Mie ellipsometric techniques to determine the particle size distribution and the optical properties of the particles in situ are presented in Secs. 3, 4, and 5. Then we show in Sec. 6 how the information can be used to determine the 3D density profile of the nanoparticles by using computed tomography or Abel inversion. Finally, from the analysis of the dust dynamics the dust parameters, such as the dust charge, and the plasma parameters, e.g. the ion and the electron densities, the plasma potential and the ion streaming velocity in the nanodusty plasma can be derived, as shown in Sec. 7. Overall, we will mainly concentrate on the capabilities, requirements and limitations of the different methods.

\section{Experimental setup and nanodusty plasma creation}

There is a variety of methods to produce nanodusty plasmas: particles can be grown directly in the plasma, or particles can be produced externally and then be immersed into the plasma. The review of Kortshagen [3] provides a recent overview about different nanoparticle sources, pressure regimes and technical applications.

Our scientific interest focuses on basic research of the dynamics of large nanoparticle clouds. For that purpose, low pressure, non-equilibrium radio frequency (rf) driven capacitively coupled parallel plate reactors are found to be ideally suited to create dense nanodusty plasmas. Fig. 2 shows the experimental setup and its dimensions.

To create the nanodusty plasma, an argon plasma is operated at a typical gas pressure of 5 to $25 \mathrm{~Pa}$ and an $\mathrm{rf}$ power of 5 to $20 \mathrm{~W}$. Admixing acetylene to the argon gas ( 5 to $30 \%$ ) leads to the growth of nanoparticles. The basic steps of particle growth with hydrocarbon molecules are shown in Fig. 3. First, different reactive precursor molecules and ions are formed from acetylene. These precursors react and build small nanoparticles $(a \approx 1 \mathrm{~nm})$. In the plasma, the small particles have fluctuating charges, a few elementary positive or negative charges. After the nucleation phase these particles suddenly coagulate to particle radii of $a \approx 10 \mathrm{~nm}$. It has been suggested [4], that the mechanism which triggers the coagulation is related

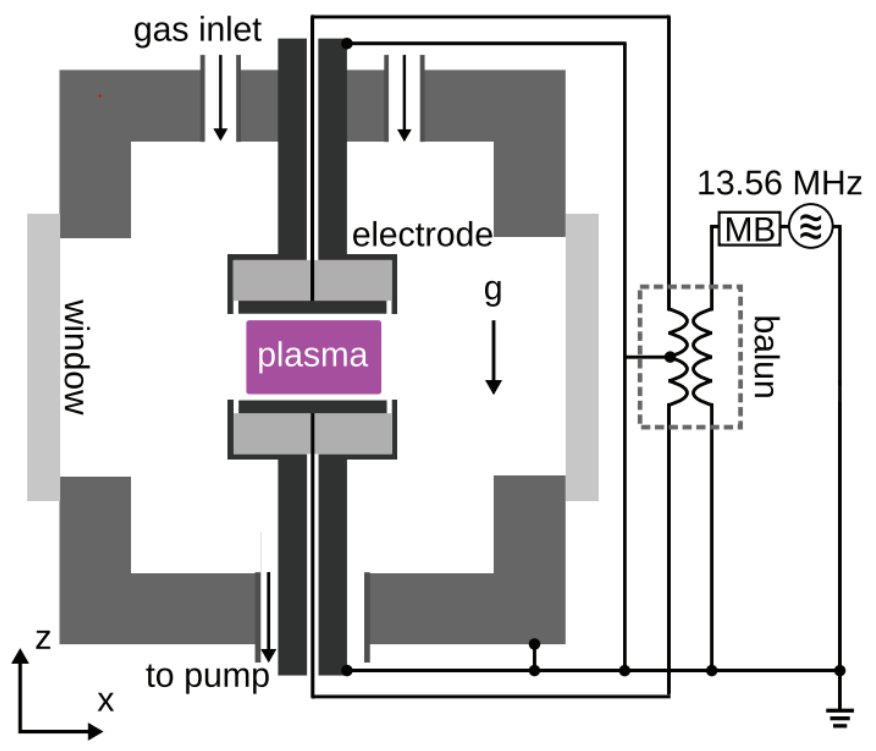

Fig. 2. Plasma chamber used for the experiments: In an stainless steel vacuum chamber a capacitively coupled $\mathrm{rf}$ discharge (at $13.56 \mathrm{MHz}$ ) is created with circular $60 \mathrm{~mm}$ diameter electrodes having a distance of $32 \mathrm{~mm}$. A balun is used to create the plasma in push-pull mode. The working gas is argon. To produce nanoparticles a small amount of acetylene is mixed to the argon.

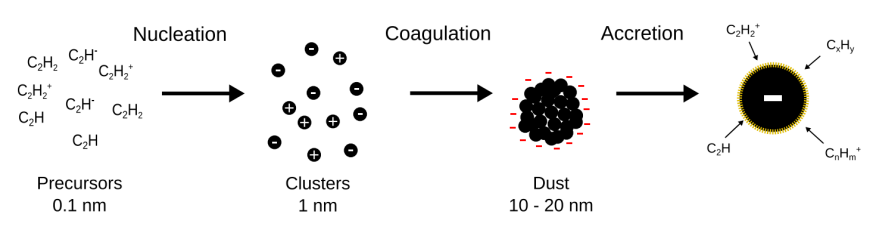

Fig. 3. Particle growth processes in an acetylene plasma. In the nucleation phase, plasma chemical processes lead to the creation of molecular precursors that nucleate to small nanoparticles. In the coagulation phase the nanoparticles suddenly coagulate to negatively charged particles which grow further by surface growth in the accretion phase.

to a critical density of the nanoparticles. During the coagulation all particles gain a high negative charge that prevents further coagulation. In the following accretion phase the particles grow further by surface growth from neutral molecules and negative ions [5]. The growth process continues as long as the particles are confined to the plasma. When the acetylene flow is switched off, the particles stop growing and a nanodusty argon plasma is formed. The particles then consist of amorphous hydrogenated carbon (a:C-H particles). On a larger timescale, due to the imperfect particle confinement and inherent plasma-etching processes, the nanoparticle density decreases and the particle size and distribution is altered, see e.g. Ref. $[6,7]$. 


\section{Nanoparticle size - Mie-Ellipsometry}

The analysis of the polarization state of light scattered by small particles is a widely used technique to determine particle sizes in different technological and physical applications $[7-10]$. The size parameter $x=2 \pi a / \lambda$ determines the light scattering regime where $\lambda$ is the wavelength of the scattered light. For a reliable size determination the size parameter should be near $x \approx 1$, which is the Mie regime of scattering. In the Rayleigh regime $(x<1)$ the scattered intensity has the well-known $1 / \lambda^{4}$ dependency. However, due to missing pronounced scattering features, this regime is not well suited for absolute size measurements of particles which grow over time, see e.g. [7] and the references therein. Affordable optics, lasers, and cameras work best in the visible spectral range. This means, that the optimal radius of the particles for Mie scattering lies around $a=80$ to $200 \mathrm{~nm}$.

One standard systems for the automated measurement of the polarization state of polarized light is a rotating compensator ellipsometer (RCE)[11]. The incident or scattered polarized light is modulated with a rotating $\lambda / 4$ plate. In the framework of the Jones formalism, the two ellipsometric angles, namely the intensity ratio $\Psi$ and the relative phase $\Delta$, are determined which describe the elliptical polarization state of the scattered light. Technically, the RCE delivers these two angles $(\Psi, \Delta)$ from a Fourier analysis of the acquired intensity signal [12]. These data are, in principle, sufficient to estimate the particle radius from the light scattered by a nanodusty plasma containing monodisperse particles.

Depending on known properties of the nanoparticles, in particular the refractive index $N=n+\mathrm{i} m$, where $n$ determines the ratio of the speed of light inside and outside of the particle and $m$ describes the absorption of light in the particle, the size of the nanoparticle can be estimated from the $(\Psi, \Delta)$ data. However, in most cases, the refractive index of a nanoparticle is not known since it is often different from the refractive index of the bulk material.

With an unknown refractive index, a single measurement $(\Psi, \Delta)$ is not sufficient to determine particle sizes. This can only be solved by adding complementary measurements to the data analysis procedure. Using the socalled kinetic Mie ellipsometry scheme, where a number of $(\Psi, \Delta)$-pairs over the whole particle growth process is acquired, both, the refractive index and the time-dependent particle radius $a(t)$ can be determined.

Figure 4 shows an example where the ellipsometric angles have been measured during particle growth [7]. The data can be excellently modeled using a constant refractive index, but an arbitrary size evolution, a technique termed CRAS-Mie (Constant Refractive index with Arbitrary Size curve) [7]. The nonlinear evolution of the particle size over time is shown in Fig. 4(c). In the presented case, the particles stop growing after about $100 \mathrm{~s}$, because the acetylene admixture has been switched off. The analysis relies on the Mie-kernel of Bohren and Huffman [13] assuming spherical particles and single scattering. It should be stressed that the absorption coefficient $m$ strongly influences the $\Delta(\Psi)$-curves, but the quality of the fit de-

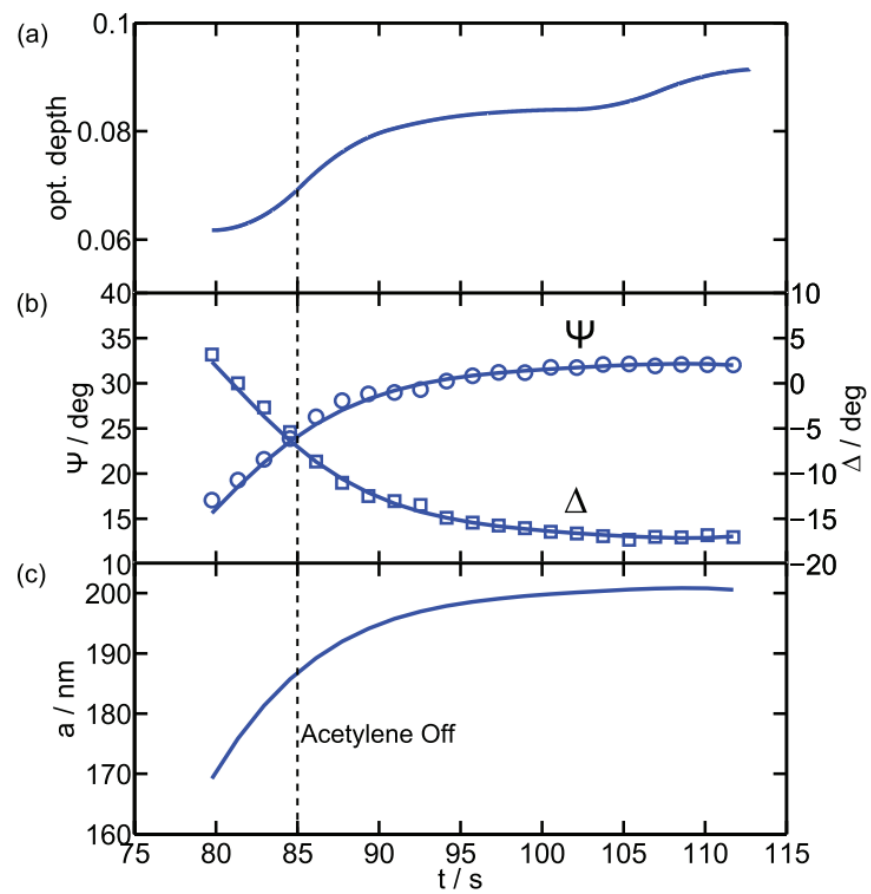

Fig. 4. CRAS-Mie has the ability to analyze data with arbitrary particle growth over time. For small optical depths the method gives a perfect fit. A nonlinear evolution of the particle radius over time was forced by switching off the acetylene flow. From [7].

pends on both, the real and the imaginary part of the refractive index. Figure 4(a) shows the optical depth $\tau$ of the nanoparticle cloud, estimated from an extinction measurement. By rule of thumb [14] single scattering can be considered to be valid for $\tau \leq 0.1$.

Depending on the confinement of the particles in the plasma, the dust density can become very high and the optical depth can easily take values $\tau>0.1$. For CRASMie and other methods, relying on single scattering in an optically thin cloud, the extracted particle size information becomes increasingly unreliable. Under these conditions radiative transfer simulations should be applied. In such Monte Carlo simulations [15] a large number of photons are followed on their way through the nanodusty plasma experiencing multiple scattering events until eventually reaching the detector. Figure 5(a) shows the multiply scattered light from a thin horizontal laser beam, entering the dust cloud from the left and reaching the $x-y$ detector plane. One sees, that through multiple scattering events, photons from all over the dust cloud are collected. In addition, Fig. 5(b) shows the optical depth calculated from simulations for typical experimental conditions with a dust density of $n_{\mathrm{d}}=4.2 \times 10^{13} \mathrm{~m}^{-3}$ [16] as well as for higher and lower densities. The parameter $\alpha$ gives the assumed dust density in units of the experimental density.

A particle size diagnostics at high optical depth has to take into account multiple scattering. Fig. 5(c) shows the experimental $(\Psi, \Delta)$ curve measured with varying dust density (circles) together with simulated curves for differ- 
(a)

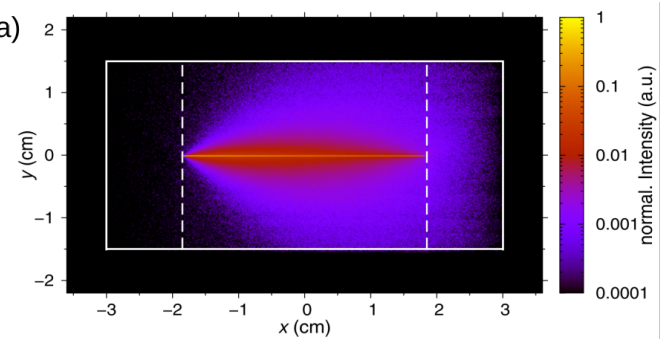

(b)

(c)
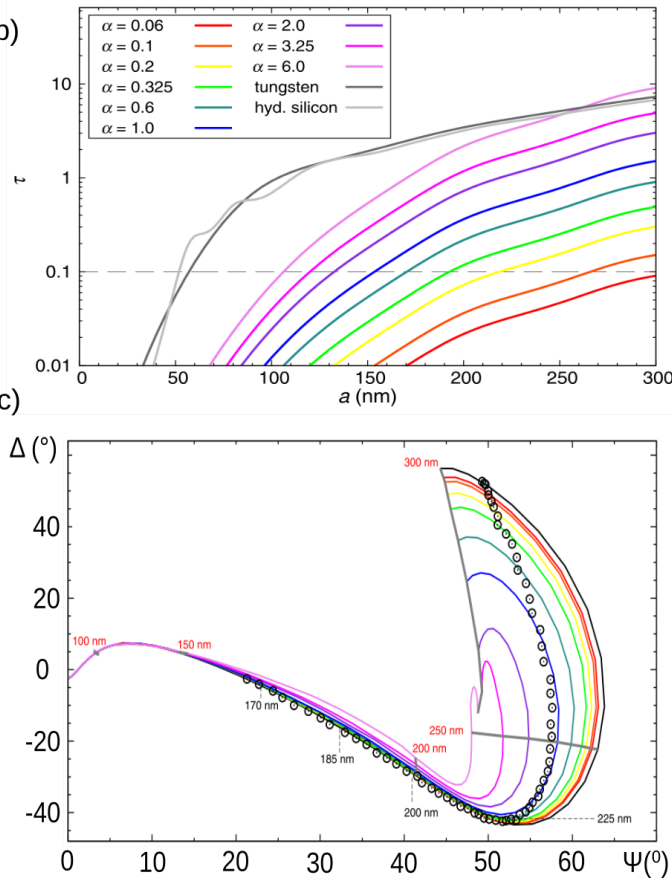

Fig. 5. (a) Light intensity distribution of a laser beam passing through the dust cloud. (b) Optical depth of the nanodust cloud. The density of the nanoparticles $\alpha$ is taken in units of the experimental value of the dust density of $n_{\mathrm{d}}=4.2 \times 10^{13} \mathrm{~m}^{-3}$ to demonstrate the effect of optical depth. (c) Simulated $\Delta(\Psi)$ plots for varied particle density with multiple scattering. The circles show an experimental curve, the simulated curve set can be used to estimate the particle density and the particle radius for optically thick particle clouds. From [15].

ent particle densities (lines). The size and density of the particles at a specific situation can now be read from the set of simulated curves.

\section{Nanoparticle size distribution - Imaging Mie-Ellipsometry}

In the last section we have presented method to extract the particle size at a single position within the cloud. However, often we face a strongly inhomogeneous cloud with a distribution of particle sizes. Then, a $2 \mathrm{D}$ ellipsometry is desirable. The situation is further complicated by the fact, that the plasma glow and the particle dynamics can lead to a partial depolarization of the light. The Jones Formalism, relying on the electric field description of electromagnetic waves, cannot describe unpolarized light, thus, the
Mueller-Stokes Formalism has to be used. In this formalism, light scattering is described using the Stokes vector

$$
\boldsymbol{S}=\left(\begin{array}{c}
I \\
Q \\
U \\
V
\end{array}\right)=\left(\begin{array}{c}
I_{0} \\
I_{\|}-I_{\perp} \\
I_{\pi / 4}-I_{-\pi / 4} \\
I_{\mathrm{R}}-I_{\mathrm{L}}
\end{array}\right),
$$

where $I=I_{0}=I_{\mathrm{p}}+I_{\mathrm{u}}$ is the total intensity consisting of the polarized part $I_{\mathrm{p}}$ and the unpolarized part $I_{\mathrm{u}}$, $I_{\|}, I_{\perp}, I_{\pi / 4}$, and $I_{-\pi / 4}$ are the linear polarized intensities measured at angles of $0^{\circ}, 90^{\circ}, 45^{\circ}$, and $135^{\circ}$ degrees with respect to the scattering plane and $I_{\mathrm{R}}$ and $I_{\mathrm{L}}$ denote rightand left-circularly polarized light. A scattering processes can be described by

$$
S_{\mathrm{s}}=\mathrm{M} S_{\mathrm{i}}
$$

where $\boldsymbol{S}_{\mathrm{i}}$ is the Stokes vector of the incoming light and $\boldsymbol{S}_{\mathrm{s}}$ that of the scattered light. The state vectors are connected via $4 \times 4$ Mueller matrices $\mathbf{M}$, see e.g. the textbook of Collett and Schaefer [12].

Different schemes exist to build a 2D imaging Mie ellipsometer. Technically straight forward is an extension of the RCE setup using a 2D laser sheet instead of a single beam and a CCD camera instead of a photo diode. First results of such a setup are presented in Sec. 4.2. The data analysis of a 2D-RCE has to face numerous challenges concerning spatial changes of the refractive index and particle size gradients. We have developed an imaging Mie scheme ("I-Mie") that requires less data analysis and that can be used in situations without strong size gradients or in situations where a qualitative spatial size analysis is sufficient. The differences between different concepts mainly lie in treatment of the spatial distribution of the degree of polarization $(\mathrm{DoP})$ of the scattered light. The $\mathrm{DoP}=I_{\mathrm{p}} / I_{0}$ is the ratio of the polarized part and the total intensity of the scattered light. For the computation of $\Delta$ and $\Psi$ the normalized Stokes vector $S \times \operatorname{DoP}=I_{\mathrm{p}}(1, q, u, v)^{T}$ has to be used, where lower case $q, u$, and $v$ indicate the normalized quantities.

\subsection{Nearly homogeneous dust cloud - global DoP}

The I-Mie setup presented in [17] uses a 2D laser stripe which is linearly polarized under an angle of $45^{\circ}$ with respect to the scattering plane, a polarizing beam splitter, and two CCD cameras measuring the intensities $I_{\|}$ and $I_{\perp}$, respectively. For fully polarized light $(\mathrm{DoP}=1)$ I-Mie directly measures the second Stokes parameter $q=$ $\left(I_{\|}-I_{\perp}\right) /\left(I_{\|}+I_{\perp}\right)$. Otherwise, the I-Mie system can be calibrated to give full spatial and temporal size information when the unpolarized part of the scattered light is time dependent but spatially constant ("global DoP" concept). Then, the Stokes component $Q$ at all spatial positions can be normalized with the DoP measured at a single spatial position (see Ref. [17] for details). For that purpose, the full RCE data is acquired at a single spatial position in addition to the CCD camera data. As the result, the measured camera data $Q$ can be calibrated to determine particle size over a 2D cross section of the nanodusty plasma. 


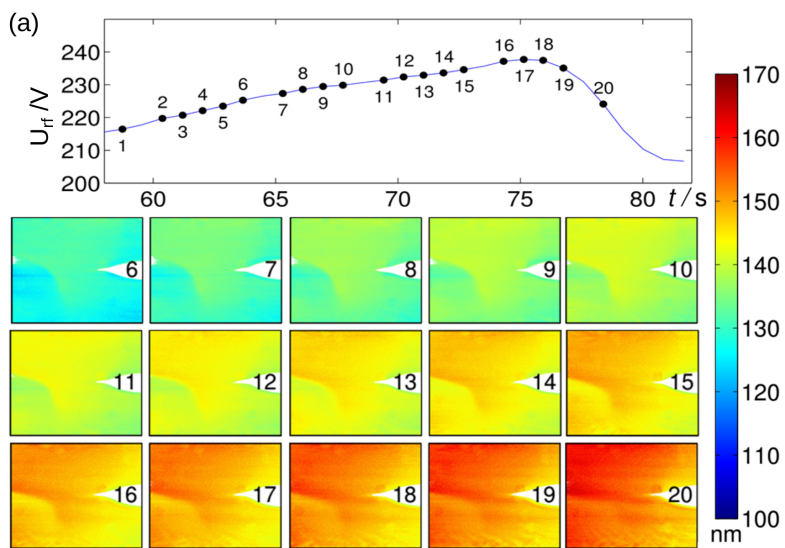

(b)

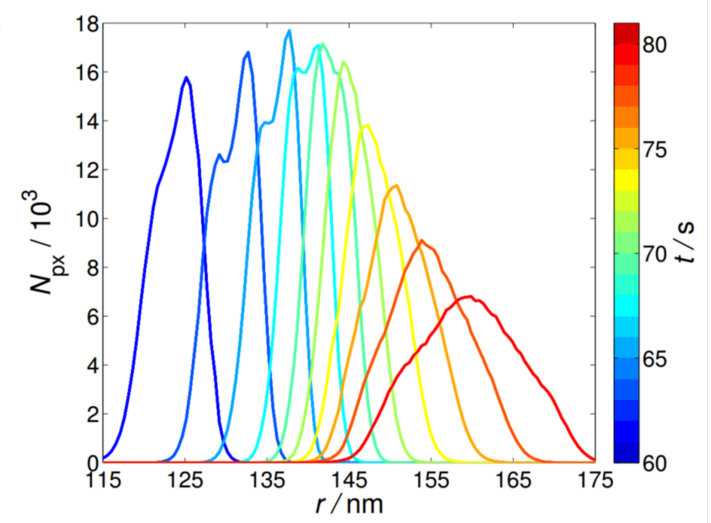

Fig. 6. (a) Using the imaging Mie technique the growth of nanoparticles can be spatio-temporally resolved. The upper graph shows the amplitude of the rf voltage during a growth cycle. The numbers show the times where snapshots of the spatial particle size distribution are taken. (b) From the size images shown in (a) the size distribution function is computed.

Figure 6(a) shows the time resolved particle radii during one growth cycle of a growth instability of particles in an argon acetylene plasma using I-Mie. The upper time series shows the change of the rf peak-to-peak voltage applied to the plasma. It changes during the particle growth because the particles change the plasma impedance and therefore the rf current through the plasma. The panels show, color-coded, the 2D-resolved particle sizes. From that the growth dynamics over time and within the cloud can be retrieved. The obtained particle size distribution within each instant of time is given in Fig. 6(b). The size distribution is strongly changing with time and seems to be different from a log-normal distribution or Gaussian distribution [18] as reported for ex-situ SEM measurements [5] or as assumed in ellipsometry or extinction data analysis procedures $[10,19]$. The presented method gives new insight into the in-situ growth dynamics of nanoparticles in a reactive plasma.

The I-Mie setup is a robust, fast and simple setup for a number of experimental situations. The full particle size calibration of I-Mie strongly relies on the measurement of the refractive index. The smallest particle size that can be measured, is mainly limited by the power and wavelength

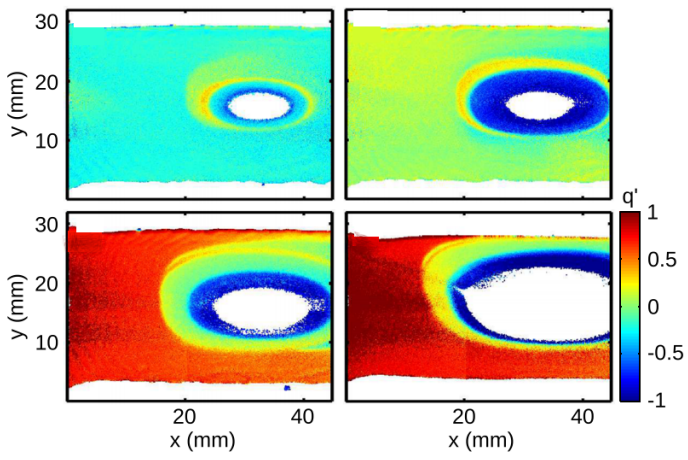

Fig. 7. Layered growth of nanoparticles can be qualitatively investigated with the simple imaging-Mie setup. $q^{\prime}=-1$ means small, $q^{\prime}=1$ means large particles. Due to a spatially varying DoP a precise size measurement is not possible in this case. From [21].

of the laser and the sensitivity of the CCD cameras. When I-Mie cannot be used, full imaging Mie ellipsometry has to be performed. There are many different concepts available, a comprehensive overview is given in Ref. [20].

\subsection{Clouds with strong density and/or size gradients}

In situations where strong size gradients exist in the particle cloud, the global DoP concept is no longer reliable. Strong gradients can be provoked by periodically switching "on" and "off" the acetylene flow [21]. This leads to a layered growth of particles. This technique was used to study the trigger mechanism for nanoparticle growth, the so called "hickup" of the void (a short contraction of the void), which indicates the start of the coagulation phase (see Fig. 5 of Ref. [21]).

Figure 7 shows different images of a growth process interrupted by a switching of the acetylene flow (see Ref. [21] for details). Although a globally constant DoP might certainly not be valid in this case, nevertheless, the I-Mie system provides an excellent qualitative diagnostics of the particle size. In contrast to $q$, which is $Q$ normalized by $I_{\mathrm{p}}$, here the parameter $q^{\prime}=\left(I_{\|}-I_{\perp}\right) /\left(I_{\|}+I_{\perp}\right)$ is used, which is $Q$ normalized with the total intensity $I_{0}$ of the scattered light (The parameter $q^{\prime}$ is identical with the polarization parameter $-P$ of Ref. [9]). It has the advantage that it is robust against dust density changes. Because $q^{\prime}$ is a multivalued function of the particle size (see Ref. [21]) during size evolution, a specific value of $q^{\prime}$ will reoccur for increasing particle size, leading to ambiguities in the size determination. This effect is not critical for a nanodusty plasma, because the ion drag force in combination with gravity eventually removes large particles from the system. In Ref. [21] a re-calibration procedure is presented, which remaps $q^{\prime}$ to a monotonous function. The methods works well for situation where a size monitor is needed and no particle dynamics with strong particle flows of different sizes are expected.

Fig. 8 shows a 2D DoP measurement for a situation, where a new growth cycle has started in the void of a grow- 

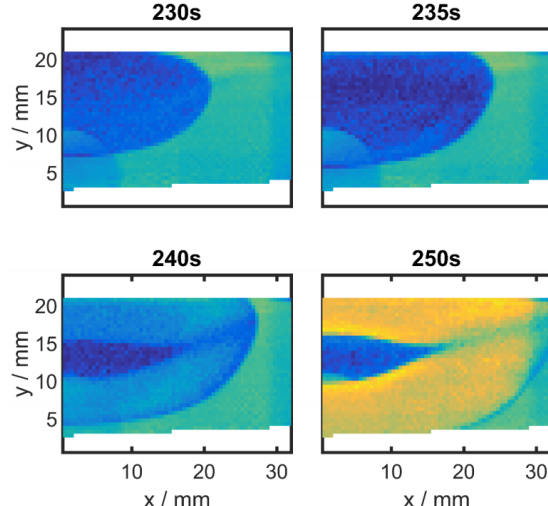

250s

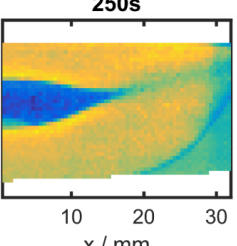

$\mathrm{x} / \mathrm{mm}$

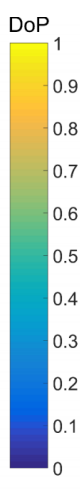

Fig. 8. Layered growth of a nanoparticle cloud leads to a spatially varying degree of polarization. The development of DoP is shown for different times of the growth cycle.

ing dust cloud (layered growth). It is immediately seen, that the DoP is not globally constant. In such a situation a quantitative analysis of the particle size demands for an imaging rotating compensator ellipsometer (I-RCE).

Currently, such an I-RCE is under development. This system uses a rotating quarter wave plate to modulate the intensity of a laser sheath in addition to a CCD camera with a linear polarizer which records the scattered light of the particle cloud. For data analysis the scattering signals of at least one period of the wave plate rotation has to be acquired. This presently limits the time resolution to $1 \mathrm{~s}$. The data analysis is quite challenging. In a first step $\Delta$ and $\Psi$ have to be extracted from the time series of the rotating wave plate (typical 80 data points at each of the camera pixels). Using the CRAS-Mie approach, a refractive index is extracted from the $\Delta(t)$ and $\Psi(t)$ datasets at each pixel position with sufficient signal-to-noise ratio. This refractive index is then used to get estimates of the particle radii $a(t, x, y)$ for each pixel $(x, y)$ over the whole video sequence. This way, a 2D diagnostic for arbitrary size development over time is established.

The main restriction of this method, as for CRASMie, currently is the assumption of a constant refractive index during growth. This assumption is plausible when a growth process is observed during stationary plasma conditions. A change of the refractive index is linked with a change of the material properties of the particle due to melting or crystallization. Such processes create or absorb energy and need time and it is unlikely that they were triggered at stationary discharge conditions.

\section{Angular-resolved Mie Ellipsometry - Spatial clustering of particles}

As an alternative method to measure particle sizes angularresolved Mie imaging can be used which exploits the fact, that the intensity of the scattered light has a strong angular variation $[14,22]$. In an experiment similar to that of Fig. 2 with $360^{\circ}$ optical access the angular resolved Mie scattering signal from a cloud of plastic (melamineformaldehyde) particles with a nominal radius of $1.775 \mu \mathrm{m}$ a)

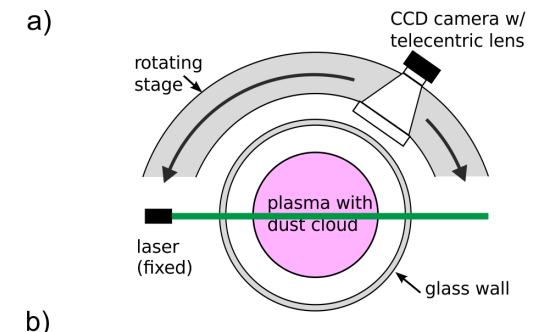

b)
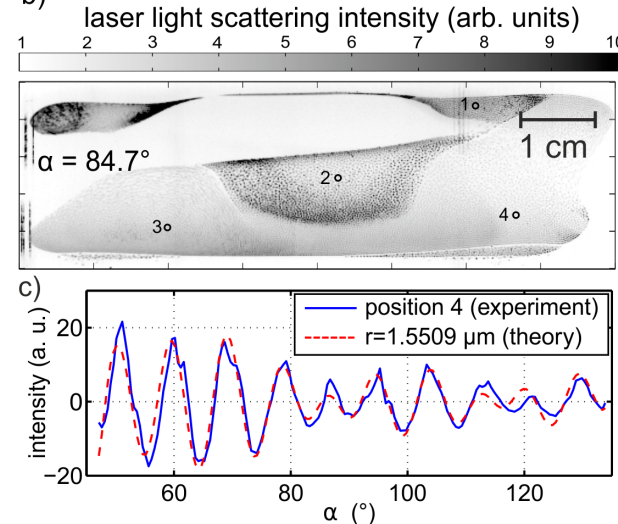

d)

1.5 1.55 radius ( $\mu \mathrm{m})$

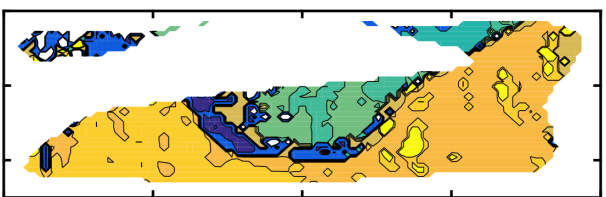

Fig. 9. (a) Scheme of the angular Mie scattering setup (top view). b) Image of the dust cloud under a scattering angle of $84.7^{\circ}$ (with respect to the laser slice). c) Angular resolved scattering intensity for position 4 indicated in b). Here, the measured intensity (solid line) is shown together with a fit from Mie theory for the corresponding size. d) Color-coded spatially resolved dust size distribution. From [23].

is measured by rotating a CCD camera with a telecentric lens around the dust cloud, see Fig. 9. There, only a thin vertical slice of the cloud is illuminated by a fixed laser beam at a wavelength of $532 \mathrm{~nm}$ and with a linear polarization in the horizontal plane.

From the image of the dust cloud under a certain scattering angle [see Fig. 9b)] already interesting features are seen: the cloud consists of segmented areas with sharp boundaries where some areas appear bright and some others appear darker. These scattered intensity drastically changes within these areas under different scattering angles. This is seen in Fig. 9c). There, the measured intensity variation over scattering angle for a certain selected point is shown together with the calculated scattering pattern from Mie theory where the refractive index $n=1.68$ for melamine-formaldehyde is used. In comparison between the measured values and the Mie fit only the particle size serves as a free parameter and the measured curves can be fitted very well to the data. The dust radii determined from the fit differ among the different regions by more than $6 \%$ which is much larger than the size dispersion given by the manufacturer. Within each bounded region, however, 

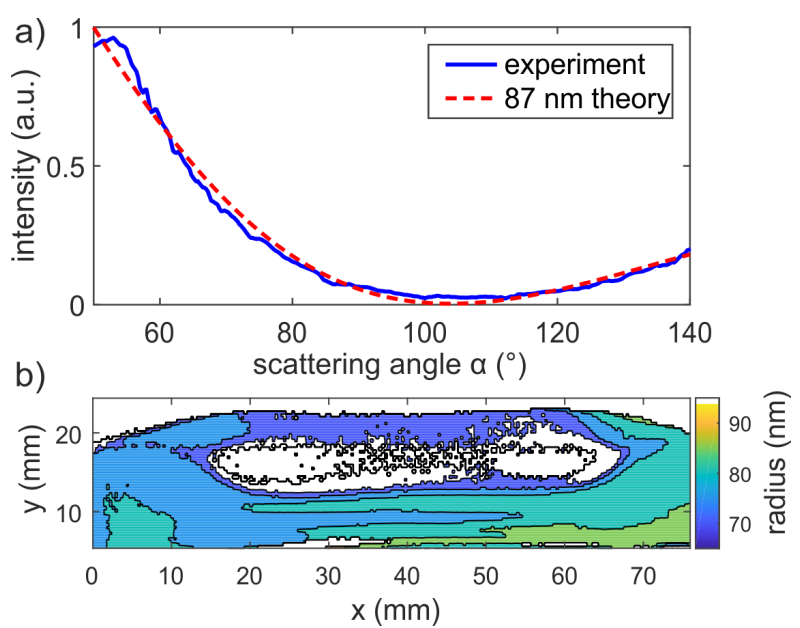

Fig. 10. a) Measured angular resolved scattering intensity for a certain position in the dust cloud (solid line) together with a fit from Mie theory for the corresponding size. b) Color-coded spatially resolved dust size distribution.

the dust particle size was found to be nearly identical. This means the dust cloud consists of patches of homogeneous particle size that are clearly separated against other regions of slightly different size [23], see Fig. 9d). Over time scales of several minutes to hours, it is seen that the particles lose mass and size due to etching processes [23] as confirmed in experiments on single particles [24].

The kinetic Mie ellipsometry in Sec. 3 overcomes the under-determined problem of particle size measurement and unknown refractive index by the combined analysis of a number of $(\Psi, \Delta)$ pairs during particle evolution. For the angular-resolved Mie scattering a simultaneous determination of particle size and refractive index using the scattering intensity at various angles will be addressed in future investigations.

The angular-resolved scattering technique can also be used for nanometer-sized dust clouds. Figure 10 shows an angular resolved scattering curve for a situation where alumina dust is trapped in a discharge. The cloud consists of particles in the radius range between 80 and $120 \mathrm{~nm}$.

\section{Spatial dust density distribution - Computed Tomography and Abel Inversion}

The full diagnostics of the nanodusty plasma requires both, the spatial particle size distribution and the spatial particle density distribution. In principle, the Mie ellipsometry can be calibrated to measure absolute intensities. For that purpose, the Rayleigh scattering signal of neutral gases (with no plasma) at different high gas pressures of about $100 \mathrm{hPa}$ to $1000 \mathrm{hPa}$ has to be measured. Using the ideal gas law, the particle density is known and the measured intensity can be calibrated. However, one of the advantages of Mie ellispometry is, that it requires only relative intensities. In addition, absolute calibration infers relatively large errors, e.g. due to parasitic light, scattered from the chamber walls, lenses, and the electrodes.
Therefore, density measurements by light extinction are preferable where the extinction of light (including absorption and scattering) passing through the nanodusty plasma is determined. There, Beer-Lambert's law can be exploited, with the optical depth $\tau(t, x, y)$

$\tau(t, x, y)=\int_{0}^{L} \pi a^{2}(t, x, y, z) Q_{\mathrm{ext}}(N, a(t, x, y, z)) n_{\mathrm{d}}(t, x, y, z) d z$,

where $Q_{\text {ext }}$ is the extinction efficiency which depends on the refractive index $N$ and the particle radius $a(t, x, y, z)$. The optical depth $\tau(t, x, y)$ is given by the integral along the line of sight ( $z$-axis). It should be stressed that the determination of a reliable density profile from the extinction measurement requires a well-known extinction efficiency $Q_{\text {ext }}$ which again relies on the refractive index and is a result of the Mie ellipsometry analysis.

Extinction measurements can be used to reconstruct the $3 \mathrm{D}$ density profile of the dust cloud. Here, we will discuss two approaches, computed tomography (CT) and Abel inversion.

Computed Tomography is the more general case, where a system consisting of a $2 \mathrm{D}$ light source (LED panel) and a CCD camera is rotated by 360 degrees around the nanodusty plasma [25]. Figure 11 shows a discharge system with $360^{\circ}$ optical access together with a scheme of the LED panel and the camera rotating around the chamber in its center. For a complete 3D dust density measurement during a full rotation of the camera/panel setup lineintegrated extinction measurements are taken at various angles. Afterwards, the 3D density distribution is retrieved via an inverse Radon transform. Due to the required rotation, the tomography measurements are relatively slow (about 10 s per rotation) and, hence, need stationary systems.

When the dust cloud can be assumed to be cylindrically symmetric, a single extinction image is sufficient to reconstruct the $3 \mathrm{D}$ particle density by means of an Abel inversion procedure [25]. There, from the line-integrated (along $z$ direction) measurement of the optical depth $\tau(x)$ along the horizontal axis $x$, the radial distribution $\tau(r)$ can be reconstructed. Repeating this for the entire image height $y$, the cylindrically symmetric distribution $\tau(r, y)$ is obtained. Ideally, one would first check with CT whether the density profile is sufficiently cylindrically symmetric and then decide to use Abel inversion. Generally, since the chamber often is cylindric with circular electrodes Abel inversion is usually justified.

Figure 11 (c, upper row) shows the 3D density profiles for a dust cloud of micrometer sized dust, created under laboratory conditions using the thermophoretic force to compensate gravity. The dust cloud has a maximum density of $4 \times 10^{11} \mathrm{~m}^{-3}$ and is clearly cylindrically symmetric. The observation of the cloud for $100 \mathrm{~min}$ reveals that the cloud loses particles mainly in the region near the bottom electrode, which is heated to produce the thermophoretic force. In contrast to the microparticle cloud, the nanoparticle cloud does not show pronounced vertical asymmetry (Fig. 11 (c, middle row)). Over 24 min the maximum density decreases from $1.4 \times 10^{13} \mathrm{~m}^{-3}$ to $0.7 \times 10^{13} \mathrm{~m}^{-3}$, while 


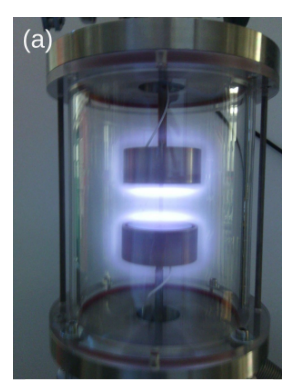

(b)

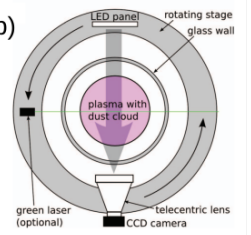

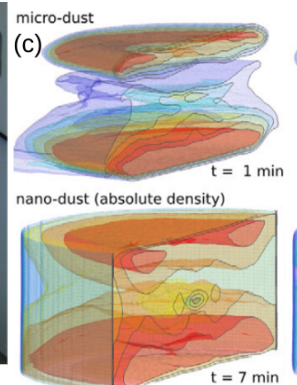

nano-dust (relative density)

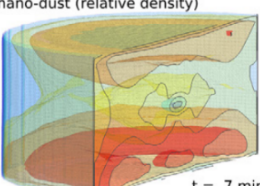

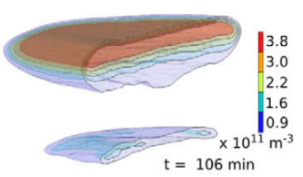
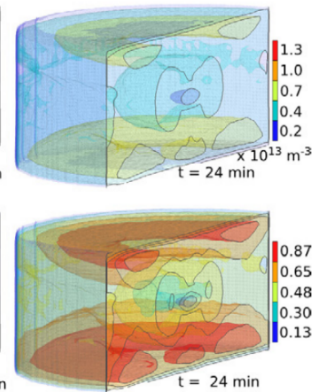

Fig. 11. (a) Plasma chamber design for $360^{\circ}$ optical access. (b) Tomography setup: An LED panel and a CCD camera are rotated around the plasma chamber. (c) Reconstructed 3D density structure of a dust cloud of micrometer sized particles (upper row) and nanometer dust (mid and bottom row). The fine structure of the dust cloud becomes visible. In contrast to the micro-dust cloud which looses particles mainly in the region near the (heated) lower electrode, the particle loss is homogeneous for the nano-dust, i.e. the spatial distribution remains unchanged. From [26].

the density profile does not change its shape. This is nicely seen in Fig. 11 (c, bottom row), which presents the same profiles, but each normalized to its maximum value. No significant change of the density distribution is seen there. In contrast to the nanodust cloud, the micrometer dust cloud requires additional external forces (thermophoresis) to confine the cloud, hence the microparticle cloud is under stress and develops a vertical density gradient.

The 3D density profile from computed tomography supports that in many cases Abel inversion is sufficiently accurate. Figure 12 shows the result of an Abel inversion of a single camera image taken without rotation of the setup revealing the high resolution that can be achieved. Due to the fact that the density profile is cylindrically symmetric, it is sufficient to take an image of only one half of the dust cloud.

\section{Dust density wave diagnostic - nanodusty plasma diagnostic}

In the preceding sections we have presented different methods to characterize the nanoparticles, their size and their density distribution with high precision. In this Section, we will address the question how the plasma changes due to the embedded nanoparticles. The plasma is mainly affected by the depletion of electrons. The dust density is of the order of $10^{13} \mathrm{~m}^{-3}$ and, hence, not much smaller than the expected ion and electron densities. Thus, the Havnes parameter in Eq. (1) can be expected to be much larger than 1.

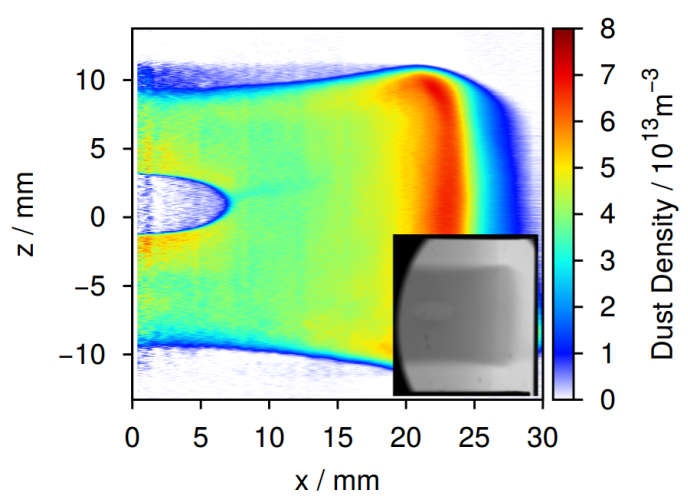

Fig. 12. Using Abel inversion, the dusty density on a $2 \mathrm{D}$ plane through the plasma center can be extracted from an extinction measurement. The inset shows the raw data before Abel inversion. A cylindrically symmetric cloud has to be assumed and the setup shown in Fig. 11(b) is used without rotation.

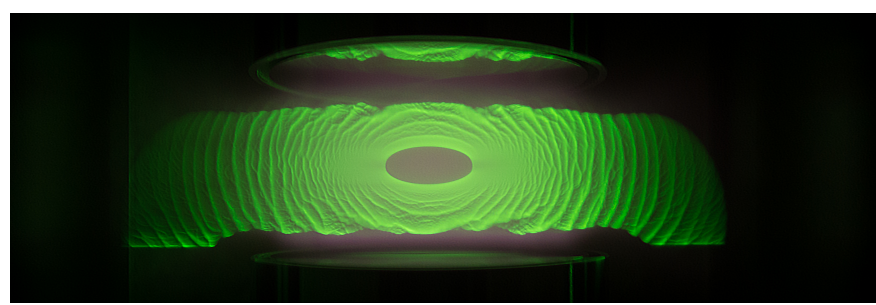

Fig. 13. Nanodusty plasma with dust density waves (DDW). The analysis of the dispersion of the DDW can be used to diagnose the nanodusty plasma.

Standard methods for the diagnostic of low temperature plasmas, like Langmuir probes, have problems, when used in dusty plasmas. In a dusty plasma a dust free region (porbe void) is created around a floating probe and the contamination of the probe is a severe problem, when a I-U-characteristic is measured [27]. In Ref. [16] we have demonstrated a non-invasive and in-situ diagnostic for the plasma parameters, which is based on the analysis of the dispersion of dust density waves (DDW), that are spontaneously excited in a nanodusty plasma. Figure 13 shows self-exited dust density waves in a dense a:C-H-particle cloud in an argon plasma. The frequency of these waves is of the order of $200 \mathrm{~Hz}$, hence to observe these waves a fast camera with sufficient frame rate has to be used. The dispersion of DDW can be described with a three fluid model for the electrons, ions and the dust $[28,29]$. The normal mode analysis of the fluid model leads to a dispersion relation

$$
0=1+\sum_{j=\mathrm{i}, \mathrm{e}, \mathrm{d}} \frac{\omega_{p j}^{2}}{k^{2} v_{T j}^{2}-\left(\omega-k v_{j 0}\right)\left(\omega-k v_{j 0}+i \nu_{j}\right)} .
$$

Here, $v_{j 0}$ is the streaming velocity, $\omega_{p j}=q_{j} \sqrt{n_{j} /\left(\epsilon_{0} m_{j}\right)}$ are the plasma frequencies, $v_{T j}=\sqrt{k_{\mathrm{B}} T_{j} / m_{j}}$ are the thermal velocities, and $\nu_{j}$ are the collision frequencies of each species. The real part $\Re(\omega)$ of eq. 5 gives the frequency and the imaginary part $\Im(\omega)$ gives the growth rate of sinusoidal waves in a homogeneous, infinite nanodusty plasma. 


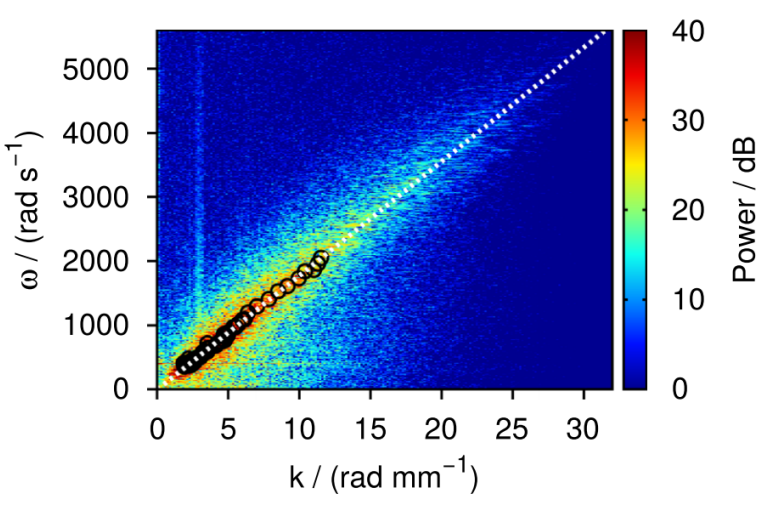

Fig. 14. Measured dispersion relation from the video sequence. The dust density wave shows an acoustic dispersion. From [16, 30].

To adopt this simple model to the experimental situation, the dispersion $\omega(k)$ of the DDW wave has to be extracted from a video of the DDW. In the following we will give a brief outline of the DDW diagnostic, see Ref. [16,30] for details.

Using a Hilbert transform of the measured spatio-temporal light intensity of the DDW the instantaneous phase and frequency can be extracted from the video. In Fig. 14 the dispersion $\omega(k)$ is presented which was extracted from the video for a small slice going radially outwards at the vertical center of the plasma. As already seen in Fig. 13, the wavelength along this axis increases and correspondingly the frequency drops. Interestingly, although the nanodusty plasma is strongly inhomogeneous, the dispersion of the wave is acoustic, i.e. phase velocity of the DDW is equal to its group velocity $\omega / k=\partial \omega / \partial k$. To obtain the plasma and dust parameters from the model solutions of the fluid model $(\omega, k)_{M}$ are sought that fit the experimental realizations $(\omega(x), k(x))_{E}$ best. Thereby we assume that the experimental situation can be approximated as a stack of small homogeneous plasma cells. The fluid model is applied to these plasmas cells, expecting that fluid mode with the largest growth rate $\Im(\omega)$ will be observed in each plasma cell. This way, the dispersion of the DDW can be described by the fluid model of a homogeneous plasma throughout the whole inhomogeneous dust cloud.

For each set $(\omega(x), k(x))_{E}$ we also get a set of plasma parameters $\left(n_{\mathrm{i}}(x), v_{\mathrm{i}}(x)\right)$ from the fitting procedure, where $v_{\mathrm{i}}$ is the ion drift velocity. Via quasi-neutrality the dust charge $q_{\mathrm{d}}(x)$ is immediately obtained from $n_{\mathrm{i}}(x)$. Using the drift approximation the electric field $E(x)$ results from $v_{\mathrm{i}}(x)$. The whole procedure needs additional input parameters, namely the electron and the ion temperature $T_{\mathrm{e}}, T_{\mathrm{i}}$, the particle radius $a$, refractive index of the particle $N$ and the dust density profile $n_{\mathrm{d}}(x)$. Here, $a$ and $N$ have to be taken from Mie ellipsometry and $n_{d}(x)$ from Abel inversion or Computed Tomography. The temperatures were assumed to be constant with $T_{\mathrm{e}}=5 \mathrm{eV}$ and $T_{\mathrm{i}}=25 \mathrm{meV}$. It was found that the profiles presented in the preceding

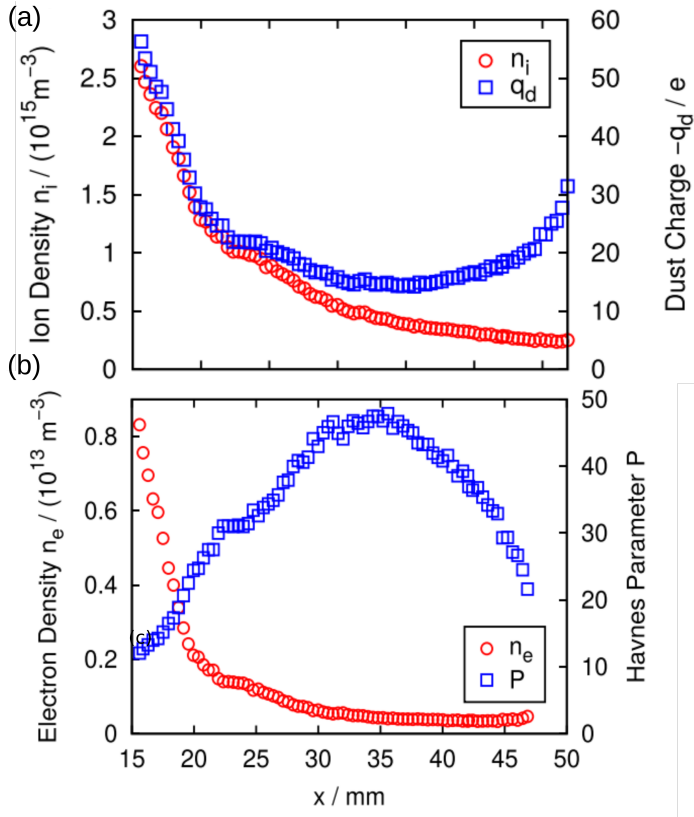

Fig. 15. Plasma parameters estimated from the DDW diagnostic. The plasma center is at the origin of the $x$-axis, which points radially outwards. (a) The ion density decreases from the center to plasma rim, the dust charge has varying values with a minimum at the rim of the electrode. (b) The electron density is more than a factor of 100 smaller than the ion density and falls continuously with increasing radial position. The strong electron depletion comes along with a high Havnes parameter, which has its maximum at the rim of the electrodes. From [16].

figures do not change much, when these temperatures are varied.

Fig. 15(a) shows the development of ion density and dust charge along the wave propagation. The ion density decreases along the radial direction $x$. In contrast, the dust charge first decreases and increases again in the region outside of the electrode $(x \approx 32 \mathrm{~mm})$. The dust charge is found to be very small compared to the OML value of $q=1600 e$. The reason for the very small measured charge clearly is the strong electron depletion. Figure 15(b) shows the profiles of electron density and Havnes parameter. The Havnes parameter increases along $x$ and reaches peak values of nearly $P=50$ at the rim of the electrode. The electron density exhibits the same profile as the ion density, but is one-hundred times smaller than the ion density, revealing a strong Havnes effect, that essentially leads to a two component dust-ion plasma.

Currently the DDW analysis was only done along a horizontal stripe, the extension to a $2 \mathrm{D}$ wave field with a particle size distribution is straightforward. It is also expected that the DDW diagnostic can be used in plasmas which do not show self-exited DDW. There, such waves can be excited and synchronized by external modulation [31]. 


\section{Conclusion}

We have presented a set of diagnostics for the full characterization of a nanodusty plasma. The methods mainly rely on optical light scattering and light absorption. Generally, these techniques are applicable to dust grown in the plasma or to dust immersed into the plasma.

The demonstrated techniques have included measurements of particle sizes, even for an unknown refractive index, spatially resolved size distributions in two dimensions as well as three-dimensional dust density distributions. Finally, the analysis of the dust dynamics allowed a deep insight into the plasma properties.

Kinetic Mie ellipsometry measures at a single point the polarization state of the scattered light. By following the evolution of the polarization state during particle growth allows to simultaneously determine the complex refractive index and the size evolution of the dust. This solves the under-determined problem to retrieve the particle size from a single polarization measurement with unknown refractive index.

For optically thick, dense dust clouds (which usually is the case for nanodusty plasmas) radiative transfer simulations help to account for multiple scattering events. From the combination of experimental scattering properties with such simulations dust density and dust size can be obtained.

Imaging Mie techniques can be used to get information about the size distribution within a dust cloud. For nearly homogeneous dust clouds, where the global DoP concept may be applied, it becomes possible to calibrate I-Mie to give the in-situ spatio-temporal evolution of the particle size distribution. In contrast to findings with ex-situ SEM analysis of the particle size, where a log-normal distribution of the particle size was found, in-situ I-Mie shows a variable shape during the particle growth phase.

Even in dust clouds with a strong particle size and/or particle density gradient, the constant DoP concept can still be used for qualitative characterization. A quantitative size analysis demands for a $2 \mathrm{D}-\mathrm{RCE}$, which is currently under development.

A different concept to measure spatially resolved dust size distribution is angle-resolved Mie scattering. This technique can be applied for dust particles of micrometer size which exhibit a strong intensity modulation with scattering angle, but also for dust in the nanometer size range. Further developments will include the simultaneous determination of dust size and refractive index.

3D particle density profiles can be determined by computed tomography methods. There the light extinction along the line of sight under various observation angles is used to reconstruct the $3 \mathrm{D}$ density profile. This requires 360 degrees optical access to the dust cloud. When cylindrical symmetry of the dust cloud can be assumed Abel inversion can be applied to measure the dust density distribution. This only requires a light extinction measurement in one direction.

From the analysis of the dust dynamics in self-excited dust-density waves the underlying dust charge and plasma parameters can be extracted. Using a fluid model to describe the excitation of dust density waves by an ion flow, the ion and electron densities as well as the dust charge can be derived. There, the inhomogeneous wave pattern is treated by a step-wise homogeneous wave field where the most unstable wave mode is calculated to fit the observed wave frequency and wave number. The quality of the DDW diagnostic rises and falls with the accurate particle size and density profile determination.

\section{Acknowledgement}

The funding by the DFG is gratefully acknowledged. The presented work is the result of 8 years research in the framework of the Collaborative Research Center, Transregio 24 "Fundamentals of Complex Plasmas" Greifswald Kiel, projects A2, A3 and A5. It would not have been possible without the technical assistance of $\mathrm{V}$. Rohwer, M. Knüppel and the IEAP workshop under the leadership of M. Brix.

\section{Author contribution statement}

FG, AM, AP, IP, CK and DB conceived and evaluated the described experiments; the experiments were prepared and performed by FG, BT, SG, CK, FW, IP, HK, who also contributed to the data analysis; FG, FK and SW conceived and evaluated the described radiative transfer simulations; the simulations were performed and analyzed by FK; the paper was written by FG and AM in collaboration with all authors; all authors contributed to the work presented here and reviewed the final text of the manuscript.

\section{References}

1. O. Havnes, C. Goertz, G. Morfill, E. Grun, W. Ip, J. Geophys. Res-Space Phys. 92, 2281 (1987)

2. I. Goertz, F. Greiner, A. Piel, Phys. Plasmas 18, 013703 (2011)

3. U.R. Kortshagen, R.M. Sankaran, R.N. Pereira, S.L. Girshick, J.J. Wu, E.S. Aydil, Chem. Rev. 116, 11061 (2016)

4. F.M.J.H. van de Wetering, R.J.C. Brooimans, S. Nijdam, J. Beckers, G.M.W. Kroesen, J. Phys. D: Appl. Phys. 48, 035204 (2015)

5. J. Berndt, E. Kovacevic, I. Stefanovic, O. Stepanovic, S.H. Hong, L. Boufendi, J. Winter, Contrib. Plasma Phys. 49, 107 (2009)

6. B. Tadsen, F. Greiner, A. Piel, Phys. Plasmas 21, 103704 (2014)

7. S. Groth, F. Greiner, B. Tadsen, A. Piel, Journal of Physics D Applied Physics 48, 465203 (2015)

8. G. Gebauer, J. Winter, New Journal of Physics 5, 38 (2003)

9. C. Hollenstein, J.L. Dorier, J. Dutta, L. Sansonnens, A.A. Howling, Plasma Sources Science Technology 3, 278 (1994)

10. Y. Hayashi, K. Tachibana, Japanese Journal of Applied Physics 33, 4208 (1994) 
11. H.G. Tompkins, A.I. Irene, eds., Handbook of Ellipsometry (William Andrew Publishing, Norwich, 2005)

12. E. Collett, B. Schaefer, Polarized Light for Scientists and Engineers (Long Branch, NY: The Polawave Group, 2012)

13. C.F. Bohren, D.R. Huffman, Absorption and Scattering of Light by Small Particles (John Wiley \& Sons, New York, 1983)

14. H.C. van de Hulst, Light Scattering by Small Particles (New York: John Wiley \& Sons, 1957)

15. F. Kirchschlager, S. Wolf, F. Greiner, S. Groth, A. Labdon, Appl. Phys. Lett. 110, 173106 (2017)

16. B. Tadsen, F. Greiner, S. Groth, A. Piel, Phys. Plasmas 22, 113701 (2015)

17. F. Greiner, J. Carstensen, N. Köhler, I. Pilch, H. Ketelsen, S. Knist, A. Piel, Plasma Sources Science Technology 21, 065005 (2012)

18. S.H. Hong, J. Winter, Journal of Applied Physics 100 (2006)

19. S. Barbosa, F.R.A. Onofri, L. Coudel, M. Wozniak, C. Montet, C. Pelc, C. Arnas, L. Boufendi, E. Kovacevic, J. Berndt et al., Journal of Plasma Physics 82 (2016)

20. J.S. Tyo, D.L. Goldstein, D.B. Chenault, J.A. Shaw, Appl. Optics 45, 5453 (2006)

21. I. Pilch, F. Greiner, J. Appl. Phys. 121, 113302 (2017)

22. W.W. Stoffels, E. Stoffels, G. Swinkels, M. Boufnichel, G. Kroesen, Phys. Rev. E 59, 2302 (1999)

23. C. Killer, M. Mulsow, A. Melzer, Plasma Sources Science Technology 24 (2015)

24. O.H. Asnaz, H. Jung, F. Greiner, A. Piel, Phys. Plasmas (submitted Jun 2017) (2017)

25. C. Killer, M. Himpel, A. Melzer, Rev. Sci. Instr. 85 (2014)

26. C. Killer, F. Greiner, S. Groth, B. Tadsen, A. Melzer, Plasma Sources Science Technology 25, 055004 (2016)

27. M. Klindworth, O. Arp, A. Piel, Rev. Sci. Instrum. 78 (2007)

28. N. Rao, P. Shukla, M. Yu, Planetary and Space Science 38, 543 (1990)

29. M. Rosenberg, Journal of Vacuum Science \& Technology A: Vacuum, Surfaces, and Films 14, 631 (1996)

30. B. Tadsen, F. Greiner, A. Piel, Phys. Plasmas 24, 033704 (2017)

31. I. Pilch, T. Reichstein, A. Piel, Physics of Plasmas 16, 123709 (2009) 